\title{
Time Domain Maxwell Equations Solved with Schwarz Waveform Relaxation Methods
}

\author{
Yves Courvoisier ${ }^{1}$ and Martin J. Gander ${ }^{2}$
}

\section{Introduction}

It is very natural to solve time dependent problems with Domain Decomposition Methods by using an implicit scheme for the time variable and then applying a classical iterative domain decomposition method at each time step. This is however not what the Schwarz Waveform Relaxation (SWR) methods do. The SWR methods are a combination of the Schwarz Domain Decomposition methods, see Schwarz [1870], and the Waveform Relaxation algorithm, see Lelarasmee et al. [1982]. Combined, one obtains a new method which decomposes the domain into subdomains on which time dependent problems are solved. Iterations are then introduced, where communication between subdomains is done at artificial interfaces along the whole time window.

This new approach has been introduced by Bjørhus [1995] for hyperbolic problems with Dirichlet boundary conditions and was analyzed for the heat equation by Gander and Stuart [1998]. Giladi and Keller [2002] analyzed this same approach applied to the advection diffusion equation with constant coefficients. For the wave equation and SWR see Gander and Halpern [2001] in which they treat the one-dimensional case with overlapping subdomains and for the $n$-dimensional case Gander and Halpern [2005], again with overlap. In this paper, we analyze for the first time the SWR algorithm applied to the time domain Maxwell equations.

\footnotetext{
University of Geneva, Section de mathématiques, Case Postale 64, 1211 Genève 4 yves.courvoisier@unige.ch · University of Geneva, Section de mathématiques, Case Postale 64, 1211 Genève 4 martin.gander@unige.ch
} 


\section{Maxwell Equations and the Schwarz Waveform Relaxation Algorithm}

The global domain $\Omega$ is decomposed into non overlapping subdomains $\tilde{\Omega}_{i}$. We denote by $\Omega_{i}$ the domain $\tilde{\Omega}_{i}$ enlarged by a band of width $\delta$ inside of $\Omega$. The part of $\partial \Omega_{i}$ in $\tilde{\Omega}_{j}$ is denoted $\Gamma_{i j}$, i.e. $\Gamma_{i j}:=\partial \Omega_{i} \cap \bar{\Omega}_{j}$. If $\Omega_{i}$ possesses a part of the boundary of the global domain $\Omega$, we denote it by $\Gamma_{i 0}:=\partial \Omega_{i} \cap \partial \Omega$. The SWR algorithm with characteristic transmission conditions for the time domain Maxwell equations is given by

$$
\left\{\begin{array}{rlrl}
-\epsilon \partial_{t} \mathbf{E}^{i, n}+\nabla \times \mathbf{H}^{i, n}-\sigma \mathbf{E}^{i, n} & =\mathbf{J}, & & \Omega_{i} \times(0, T), \\
\mu \partial_{t} \mathbf{H}^{i, n}+\nabla \times \mathbf{E}^{i, n} & =0, & & \Omega_{i} \times(0, T), \\
\mathcal{B}_{\mathbf{n}_{i}}\left(\mathbf{E}^{i, n}, \mathbf{H}^{i, n}\right) & =0, & & \Gamma_{i 0} \times(0, T), \\
\left(\mathbf{E}^{i, n}, \mathbf{H}^{i, n}\right)(\mathbf{x}, 0) & =\left(\mathbf{E}_{0}, \mathbf{H}_{0}\right), & & \Omega_{i}, \\
\mathcal{B}_{\mathbf{n}_{i}}\left(\mathbf{E}^{i, n}, \mathbf{H}^{i, n}\right) & =\mathcal{B}_{\mathbf{n}_{i}}\left(\mathbf{E}^{j, n-1}, \mathbf{H}^{j, n-1}\right), & \Gamma_{i j} \times(0, T),
\end{array}\right.
$$

where $\epsilon$ is the electric permittivity, $\mu$ the magnetic permeability and $\sigma$ the conductivity. The indices $i$ and $j$, always different, range over the indices of all subdomains, i.e. $i, j \in\{1,2, \ldots, I\}$ with $i \neq j$ and $I$ being the number of subdomains. In the algorithm $\mathbf{n}_{i}$ is the unit outward normal vector to $\Omega_{i}$. The impedance

$$
\mathcal{B}_{\mathbf{n}}(\mathbf{E}, \mathbf{H}):=\frac{\mathbf{E}}{Z} \times \mathbf{n}+\mathbf{n} \times(\mathbf{H} \times \mathbf{n}),
$$

plays the role of the Dirichlet value for this hyperbolic system (Dolean et al. [2009]) and corresponds to the inward characteristic variables of the Maxwell equations. The last line of (1), which is called the characteristic transmission condition, establishes how the subdomains communicate with each other.

\section{Convergence in a Finite Number of Steps}

From now on, we restrict our analysis to the specific situation where $\Omega=\mathbb{R}^{3}$ which is subdivided into two subdomains

$$
\Omega_{1}=(-\infty, L] \times \mathbb{R}^{2}, \quad \Omega_{2}=[0,+\infty) \times \mathbb{R}^{2} .
$$

The artificial boundaries are therefore given by $\Gamma_{12}=\{L\} \times \mathbb{R}^{2}$ and $\Gamma_{21}=$ $\{0\} \times \mathbb{R}^{2}$ with an overlap of width $L$. We also choose the coefficients $\epsilon, \mu$ and $\sigma$ to be constant.

Maxwell equations describe the motion of electromagnetic waves which propagate at finite speed, namely the speed of light in the vacuum. This fact has been proven for a broad class of hyperbolic systems, see for instance Rauch [2005]; the Maxwell equations are simply one such example. The speed of propagation is given by $c:=1 / \sqrt{\epsilon \mu}$, which is constant. 
Remark 1. The next result also holds when the coefficients are non constant and with a domain $\Omega$ decomposed into many subdomains $\Omega_{i}$ having a more complicated geometry and non constant overlap width.

Proposition 1 (Convergence in a finite number of steps). The $S W R$ algorithm (1) for two subdomains defined in (2) with overlap $L$ converges as soon as the number of iterations $n$ satisfies

$$
n>\frac{T c}{L},
$$

where $T$ is the length of the time interval and $c=1 / \sqrt{\epsilon \mu}$ is the speed of propagation.

Proof. The Maxwell equations are linear and thus allow us to restrict our attention to the error equations, i.e. (1) where $\mathbf{J}=0$ and $\left(\mathbf{E}_{0}, \mathbf{H}_{0}\right)=0$. We prove in the following that for $t<t_{n}:=n \frac{L}{c}$,

$$
\operatorname{Supp}\left(\mathbf{E}^{i, n+1}, \mathbf{H}^{i, n+1}\right)(t)=\emptyset, \quad t<t_{n} .
$$

The error of the Maxwell equations is non-zero at iteration one only because the initial guesses $\left(\mathbf{E}^{i, 0}, \mathbf{H}^{i, 0}\right)$ are non-zero on the artificial boundaries $\Gamma_{i j}$. The speed of propagation is finite and thus the error propagates from the artificial boundaries inside the domain $\Omega_{i}$. For the first iteration we have that

$$
\operatorname{Supp}\left(\mathbf{E}^{i, 1}, \mathbf{H}^{i, 1}\right)(t) \subset\left\{\mathbf{x} \in \Omega_{i} \mid \operatorname{dist}\left(x, \Gamma_{i j}\right)<t c, j \neq i, j \in\{1,2\}\right\},
$$

since after a time $t$, the electromagnetic wave can only have propagated on a distance $t c$ from the artificial boundaries. The overlap is of width $L$, hence $\left(\mathbf{E}^{1,1}, \mathbf{H}^{1,1}\right)(0, y, z, t)$ and $\left(\mathbf{E}^{2,1}, \mathbf{H}^{2,1}\right)(L, y, z, t)$ are zero unless $t c>L$, i.e. unless the time is greater or equal to $t_{1}:=\frac{L}{c}$.

For the next iteration we have that the trace of $\left(\mathbf{E}^{1,1}, \mathbf{H}^{1,1}\right)$ at $\Gamma_{21}$ and $\left(\mathbf{E}^{2,1}, \mathbf{H}^{2,1}\right)$ at $\Gamma_{12}$ are zero for times $t<t_{1}$, i.e. $B_{\mathbf{n}_{i}}\left(\mathbf{E}^{j, n-1}, \mathbf{H}^{j, n-1}\right)=0$ at $\Gamma_{i j}$ for $n=2$ and $t<t_{1}$. Therefore, when solving for $\left(\mathbf{E}^{i, 2}, \mathbf{H}^{i, 2}\right)$ we see that for $t<t_{1}$, we have zero boundary conditions and zero initial condition, hence

$$
\left(\mathbf{E}^{i, 2}, \mathbf{H}^{i, 2}\right)(\mathbf{x}, t)=0, \quad \text { for } t<t_{1} .
$$

For times $t>t_{1}$, we have a similar result as for the first iteration, namely

$$
\operatorname{Supp}\left(\mathbf{E}^{i, 2}, \mathbf{H}^{i, 2}\right)(t) \subset\left\{\mathbf{x} \in \Omega_{i} \mid \operatorname{dist}\left(x, \Gamma_{i j}\right)<\left(t-t_{1}\right) c, j \neq i, j \in\{1,2\}\right\} .
$$

We define $t_{2}:=\frac{L}{c}+t_{1}=2 t_{1}$, such that $\operatorname{Supp}\left(\mathbf{E}^{i, 2}, \mathbf{H}^{i, 2}\right)(t)=0$ on $\Gamma_{j i}$ for $t<t_{2}$. And so forth for the following iterations, which proves (3).

Hence, if $T$, the length of the time window, is finite and $t_{n}:=n \frac{L}{c}>T$, the solution $\left(\mathbf{E}^{i, n+1}, \mathbf{H}^{i, n+1}\right)$ is zero and the algorithm has converged. 


\section{Convergence of the SWR Algorithm}

Under the same setting (2) as in previous section, we prove that the SWR algorithm (1) also has a contraction factor.

Theorem 1. The convergence factor of the classical Schwarz Waveform Relaxation algorithm (1) in the frequency domain with domain decomposition (2) is given by

$$
\rho\left(s, k_{y}, k_{z}, L, \sigma\right)=\left|\frac{\sqrt{|\mathbf{k}|^{2}+\mu s^{2} \epsilon+\mu s \sigma}-s \sqrt{\mu \epsilon}}{\sqrt{|\mathbf{k}|^{2}+\mu s^{2} \epsilon+\mu s \sigma}+s \sqrt{\mu \epsilon}} e^{-L \sqrt{|\mathbf{k}|^{2}+\mu s^{2} \epsilon+\mu s \sigma}}\right|,
$$

where $s$ is the Laplace variable, $\Re(s) \geq 0$, and $|\mathbf{k}|^{2}=k_{y}^{2}+k_{z}^{2}$ is the sum of the squares of the Fourier frequencies in the $y$ and $z$ directions.

Proof. We consider the error equations for which $\mathbf{J}$ and the initial condition are zero. We first apply the Laplace transform to (1) which transforms the time $t$ into a complex frequency $s$ with $\Re(s) \geq 0$ and transforms the derivative with respect to $t$ into a multiplication by $s$. Then we apply a Fourier transform in the $y$ and $z$ directions and obtain,

$$
\frac{\partial}{\partial x}\left[\begin{array}{c}
\check{E}_{2} \\
\check{E}_{3} \\
\check{H}_{2} \\
\check{H}_{3}
\end{array}\right]+\left[\begin{array}{cccc}
0 & 0 & -\frac{k_{y} k_{z}}{\epsilon s+\sigma} & \frac{k_{y}^{2}}{\epsilon s+\sigma}+\mu s \\
0 & 0 & -\frac{k_{z}^{2}}{\epsilon s+\sigma}-\mu s & \frac{k_{y} k_{z}}{\epsilon s+\sigma} \\
\frac{k_{y} k_{z}}{\mu s} & -\frac{k_{y}^{2}}{\mu s}-(\epsilon s+\sigma) & 0 & 0 \\
\frac{k_{z}^{2}}{\mu s}+\epsilon s+\sigma & -\frac{k_{y} k_{z}}{\mu s} & 0 & 0
\end{array}\right]\left[\begin{array}{c}
\check{E}_{2} \\
\check{E}_{3} \\
\check{H}_{2} \\
\check{H}_{3}
\end{array}\right]=0
$$

For components $\check{E}_{1}$ and $\check{H}_{1}$, we have two algebraic equations

$$
\begin{aligned}
-\epsilon s \check{E}_{1}+i k_{y} \check{H}_{3}-i k_{z} \check{H}_{2}-\sigma \check{E}_{1} & =0, \\
\mu s \check{H}_{1}+i k_{y} \check{E}_{3}-i k_{z} \check{E}_{2} & =0 .
\end{aligned}
$$

The solution of (4) is given by a linear combination of the eigenvectors times an exponential of the corresponding eigenvalue,

$$
\begin{aligned}
& \left(\check{E}_{2}^{1, n}, \check{E}_{3}^{1, n}, \check{H}_{2}^{1, n}, \check{H}_{3}^{1, n}\right)^{T}=\left(\alpha_{1}^{n} \mathbf{v}_{1}+\alpha_{2}^{n} \mathbf{v}_{2}\right) e^{-\lambda(x-L)}+\left(\alpha_{3}^{n} \mathbf{v}_{3}+\alpha_{4}^{n} \mathbf{v}_{4}\right) e^{\lambda(x-L)} \\
& \left(\check{E}_{2}^{2, n}, \check{E}_{3}^{2, n}, \check{H}_{2}^{2, n}, \check{H}_{3}^{2, n}\right)^{T}=\left(\beta_{1}^{n} \mathbf{v}_{1}+\beta_{2}^{n} \mathbf{v}_{2}\right) e^{-\lambda x}+\left(\beta_{3}^{n} \mathbf{v}_{3}+\beta_{4}^{n} \mathbf{v}_{4}\right) e^{\lambda x}
\end{aligned}
$$

where $\lambda=\sqrt{|k|^{2}+\mu s^{2} \epsilon+\mu s \sigma}$ and the eigenvalues are $\lambda_{1,2}=-\lambda$ and $\lambda_{3,4}=$ $\lambda$. The corresponding eigenvectors are 


$$
\begin{aligned}
& \mathbf{v}_{1}=\left(\begin{array}{c}
\frac{k_{y} k_{z}}{\lambda(\epsilon s+\sigma)} \\
\frac{k_{z}^{2}+\mu s^{2} \epsilon+\mu s \sigma}{\lambda(\epsilon s+\sigma)} \\
1 \\
0
\end{array}\right), \mathbf{v}_{2}=\left(\begin{array}{c}
-\frac{k_{y}^{2}+\mu s^{2} \epsilon+\mu s \sigma}{\lambda(\epsilon s+\sigma)} \\
-\frac{k_{y} k_{z}}{\lambda(\epsilon s+\sigma)} \\
0 \\
1
\end{array}\right) \\
& \mathbf{v}_{3}=\left(\begin{array}{c}
-\frac{k_{y} k_{z}}{\lambda(\epsilon s+\sigma)} \\
-\frac{k_{z}^{2}+\mu s^{2} \epsilon+\mu s \sigma}{\lambda(\epsilon s+\sigma)} \\
1 \\
0
\end{array}\right), \mathbf{v}_{4}=\left(\begin{array}{c}
\frac{k_{y}^{2}+\mu s^{2} \epsilon+\mu s \sigma}{\lambda(\epsilon s+\sigma)} \\
\frac{k_{y} k_{z}}{\lambda(\epsilon s+\sigma)} \\
0 \\
1
\end{array}\right) \text {. }
\end{aligned}
$$

The speed of propagation is finite. The wave of the error equations propagates starting from the interfaces. Therefore, no wave is coming from the infinite boundary and then the growing exponential term of (5) is not present in the solution, i.e. $\alpha_{1}=\alpha_{2}=\beta_{3}=\beta_{4}=0$. Hence,

$$
\begin{aligned}
& \left(\check{E}_{2}^{1, n}, \check{E}_{3}^{1, n}, \check{H}_{2}^{1, n}, \check{H}_{3}^{1, n}\right)^{T}=\left(\alpha_{3}^{n} \mathbf{v}_{3}+\alpha_{4}^{n} \mathbf{v}_{4}\right) e^{\lambda(x-L)} \\
& \left(\check{E}_{2}^{2, n}, \check{E}_{3}^{2, n}, \check{H}_{2}^{2, n}, \check{H}_{3}^{2, n}\right)^{T}=\left(\beta_{1}^{n} \mathbf{v}_{1}+\beta_{2}^{n} \mathbf{v}_{2}\right) e^{-\lambda x}
\end{aligned}
$$

To determine the values of $\alpha_{i}$ and $\beta_{i}$, we need to use the transmission conditions. They are, for the first subdomain, $\mathcal{B}_{\mathbf{n}}\left(\check{\mathbf{E}}^{1, n}, \check{\mathbf{H}}^{1, n}\right)=\mathcal{B}_{\mathbf{n}}\left(\check{\mathbf{E}}^{2, n-1}, \check{\mathbf{H}}^{2, n-1}\right)$ with $\mathbf{n}=(1,0,0)^{T}$, i.e.

$$
\left[\begin{array}{c}
\frac{1}{Z} \check{E}_{3}^{1, n}+\check{H}_{2}^{1, n} \\
-\frac{1}{Z} \check{E}_{2}^{1, n}+\check{H}_{3}^{1, n}
\end{array}\right]=\left[\begin{array}{c}
\frac{1}{Z} \check{E}_{3}^{2, n-1}+\check{H}_{2}^{2, n-1} \\
-\frac{1}{Z} \check{E}_{2}^{2, n-1}+\check{H}_{3}^{2, n-1} \cdot
\end{array}\right]
$$

We substitute the values of the electric and magnetic fields by their values given in (7). This gives an equation relating $\boldsymbol{\alpha}^{n}=\left(\alpha_{3}^{n}, \alpha_{4}^{n}\right)^{T}$ and $\boldsymbol{\beta}^{n}=$ $\left(\beta_{1}^{n}, \beta_{2}^{n}\right)^{T}$,

$$
A_{1} \boldsymbol{\alpha}^{n}=A_{2} e^{-\lambda L} \boldsymbol{\beta}^{n-1},
$$

where matrices $A_{1}$ and $A_{2}$ are given by

$$
\begin{aligned}
& A_{1}=\left[\begin{array}{cc}
-\left(k_{z}^{2}+\mu s^{2} \epsilon+\mu s \sigma\right)+Z \lambda(\epsilon s+\sigma) & k_{y} k_{z} \\
k_{y} k_{z} & -\left(k_{y}^{2}+\mu s^{2} \epsilon+\mu s \sigma\right)+Z \lambda(\epsilon s+\sigma)
\end{array}\right] \\
& A_{2}=\left[\begin{array}{cc}
k_{z}^{2}+\mu s^{2} \epsilon+\mu s \sigma+Z \lambda(\epsilon s+\sigma) & -k_{y} k_{z} \\
-k_{y} k_{z} & k_{y}^{2}+\mu s^{2} \epsilon+\mu s \sigma+Z \lambda(\epsilon s+\sigma)
\end{array}\right]
\end{aligned}
$$

We do the same computations for the second subdomain for which we have the transmission conditions $\mathcal{B}_{-\mathbf{n}}\left(\hat{\mathbf{E}}^{2, n}, \hat{\mathbf{H}}^{2, n}\right)=\mathcal{B}_{-\mathbf{n}}\left(\hat{\mathbf{E}}^{1, n-1}, \hat{\mathbf{H}}^{1, n-1}\right)$, and obtain

$$
A_{1} \boldsymbol{\beta}^{n}=A_{2} e^{-\lambda L} \boldsymbol{\alpha}^{n-1} .
$$

We isolate $\boldsymbol{\alpha}^{n}$ and $\boldsymbol{\beta}^{n}$ in (8) and (10) and iterate one more time to obtain

$$
\boldsymbol{\alpha}^{n}=\left(A_{1}^{-1} A_{2}\right)^{2} e^{-2 \lambda L} \boldsymbol{\alpha}^{n-2}, \boldsymbol{\beta}^{n}=\left(A_{1}^{-1} A_{2}\right)^{2} e^{-2 \lambda L} \boldsymbol{\beta}^{n-2} .
$$


The parameters $\boldsymbol{\alpha}^{n}$ and $\boldsymbol{\beta}^{n}$ characterize completely the solution of (4), therefore the effective contraction factor after two iterations is given by the spectral radius of $\left(A_{1}^{-1} A_{2}\right)^{2} e^{-2 \lambda L}$. This matrix has eigenvalues

$$
\nu_{1}:=\left(\frac{\lambda-s \sqrt{\epsilon \mu}}{\lambda+s \sqrt{\epsilon \mu}}\right)^{2} e^{-2 \lambda L}, \quad \nu_{2}:=\left(\frac{\lambda-s \sqrt{\epsilon \mu}-Z \sigma}{\lambda+s \sqrt{\epsilon \mu}+Z \sigma}\right)^{2} e^{-2 \lambda L} .
$$

The largest eigenvalue in modulus is given by the first one which concludes the proof.

Corollary 1. The SWR algorithm (1) with non-zero conductivity, $\sigma>0$, converges in the $L^{2}$ norm, i.e. if we denote by $e^{i, n}:=\left(E_{2}^{i, n}, E_{3}^{i, n}, H_{2}^{i, n}, H_{3}^{i, n}\right)$, then

$$
\left\|e^{i, n}\left(\Gamma_{i j}, t\right)\right\|_{2} \longrightarrow 0 \quad(n \rightarrow+\infty),
$$

where $\Gamma_{i j}$ is defined in (2) and $\|\cdot\|_{2}$ denotes the norm in $L^{2}\left(0, T ; L^{2}\left(\mathbb{R}^{2}\right)\right)$ componentwise.

Proof. We use the notation $\check{e}^{i, n}=\left(\check{E}_{2}^{i, n}, \check{E}_{3}^{i, n}, \check{H}_{2}^{i, n}, \check{H}_{3}^{i, n}\right)$ for the solution in the Fourier Laplace variables. From relations (11) with the notation $R:=$ $A_{1}^{-1} A_{2} e^{-\lambda L}$ and iterating $2 n$ times we obtain

$$
\boldsymbol{\alpha}^{2 n}=R^{2 n} \boldsymbol{\alpha}^{0}, \quad \boldsymbol{\beta}^{2 n}=R^{2 n} \boldsymbol{\beta}^{0} .
$$

The matrix $R$ has eigenvalues $\nu_{1}$ and $\nu_{2}$ and therefore can be diagonalized using the matrix of eigenvectors $S$, i.e. $D=S^{-1} R S$. The following argument, for the first subdomain $\Omega_{1}$, is similar also for the second one.

We define $\gamma^{n}:=S^{-1} \boldsymbol{\alpha}^{n}$ for all $n=0,1, \ldots$, and from (7) we can reconstruct the solution of $\check{e}^{1,2 n}$ from the initial iterate,

$$
\begin{aligned}
\check{e}^{1,2 n}\left(x, k_{y}, k_{z}, s\right)=e^{\lambda(x-L)}\left[\mathbf{v}_{3} \mathbf{v}_{4}\right] R^{2 n} \boldsymbol{\alpha}^{0} & =e^{\lambda(x-L)}\left[\mathbf{v}_{3} \mathbf{v}_{4}\right] S S^{-1} R^{2 n} S \boldsymbol{\gamma}^{0} \\
& =e^{\lambda(x-L)}\left[\mathbf{v}_{3} \mathbf{v}_{4}\right] S D^{2 n} \boldsymbol{\gamma}^{0} .
\end{aligned}
$$

The diagonal matrix is of the form $D=\operatorname{diag}\left(\nu_{1}, \nu_{2}\right)$, hence we obtain a new form for the solution evaluated at $x=L$,

$$
\check{e}^{1,2 n}\left(L, k_{y}, k_{z}, s\right)=\nu_{1}^{2 n} \gamma_{1}^{0} \mathbf{w}_{1}+\nu_{2}^{2 n} \gamma_{2}^{0} \mathbf{w}_{2},
$$

where $\left[\mathbf{w}_{1} \mathbf{w}_{2}\right]:=\left[\mathbf{v}_{3} \mathbf{v}_{4}\right] S$.

Finally Theorem 7.23 of Rudin [1973] shows that the limit $\check{e}^{i, n}\left(L, k_{y}, k_{z}, s\right)$ when $s=\xi+i \omega \rightarrow i \omega$ is the Fourier transform of $e^{i, n}$ in the $y, z$ and $t$ variables. Therefore the Plancherel theorem applies and

$$
\left\|e^{i, n}(L, y, z, t)\right\|_{2}=\left\|\check{e}^{i, n}\left(L, k_{y}, k_{z}, i \omega\right)\right\|_{2},
$$

which implies by (12)

$$
\left\|e^{i, n}(L, y, z, t)\right\|_{2}=\left\|\nu_{1}^{2 n} \gamma_{1}^{0} \mathbf{w}_{1}+\nu_{2}^{2 n} \gamma_{2}^{0} \mathbf{w}_{2}\right\|_{2}
$$


By the dominated convergence theorem we can insert the limit, when $n$ goes to infinity, into the norm and, since $\lim _{n \rightarrow \infty} \nu_{i}$ is almost everywhere zero for $i=1,2$, it concludes the proof.

\section{Numerical Experiments}

For this section we restrict the geometry of the global domain to $\Omega=[0,1]^{3}$ and to subdomains

$$
\Omega_{1}=\left[0, \frac{1}{2}+2 \Delta x\right] \times[0,1] \times[0,1], \quad \Omega_{2}=\left[\frac{1}{2}, 1\right] \times[0,1] \times[0,1],
$$

where $\Delta x$ is the spatial mesh size in the direction $x$. We consider a time window of length $T=1$. The parameters $\epsilon, \mu$ and $\sigma$ are constant and equal to one. On the physical domain we set boundary conditions for perfectly conducting medium.

The discretization is done with the Yee scheme which is explicit in time. We set a global grid on the whole domain $\Omega$ having 24 grid points in each direction $x, y$ and $z$. The overlap is of 2 mesh points. The number of grid points for the time variable is $N=144$ which guarantees that the CFL condition is satisfied. Since the domain is bounded, only a finite number of discrete frequencies are possible. Since the domain is of width one, the minimum frequency in space is given by $k_{\min }=\pi$ and the maximum by $k_{\max }=\frac{\pi}{\Delta y}$. Equivalently for the time frequencies we have $\omega_{\max }=\frac{\pi}{\Delta t}$. Since there is no finite value imposed, we take $\omega_{\min }=\frac{\pi}{2 T}=\frac{\pi}{2}$. The discrete frequencies are therefore given by

$$
k_{y}, k_{z} \in\left\{\pi, 2 \pi, \ldots, \frac{\pi}{\Delta y}\right\}, \quad \omega \in\left\{\frac{\pi}{2}, \pi, \ldots, \frac{\pi}{\Delta t}\right\} .
$$

From Corollary 1 we have that

$$
\left\|e^{i, n}(L, y, z, t)\right\|_{2} \leq C \max _{\left(k_{y}, k_{z}, \omega\right)}\left|\nu_{1}\right|^{n}
$$

where the constant $C$ is the maximum over all frequencies of $\| \gamma_{1}^{0} \mathbf{w}_{1}+$ $\frac{\nu_{2}}{\nu_{1}} \gamma_{2}^{0} \mathbf{w}_{2} \|_{2}$. We also expect the solution to converge in a finite number of iterations as shown in Figure 1.

\section{References}

Morten Bjørhus. On Domain Decomposition, Subdomain Iteration and Waveform Relaxation. PhD thesis, University of Trondheim, Norway, 1995. 


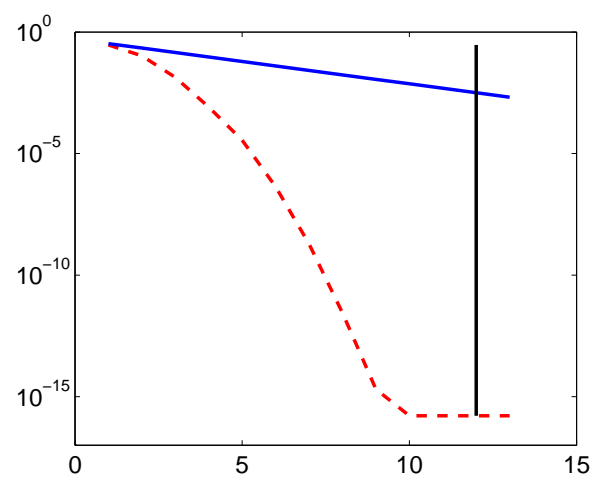

Fig. 1 The plain blue line is the upper bound in (13), and the dashed line is the error $\left\|E_{2}^{1, n}\right\|$ in the $L^{2}$ norm evaluated at the interface $x=b$ with respect to the iterations. The error converges before the relation of Proposition 1 is satisfied (vertical line).

V. Dolean, M.J. Gander, and L. Gerardo-Giorda. Optimized Schwarz methods for Maxwell's equations. SIAM Journal on Scientific Computing, 31 (3):2193-2213, 2009.

Martin J. Gander and Laurence Halpern. Méthodes de décomposition de domaines pour l'équation des ondes en dimension 1. C. R. Acad. Sci. Paris Sér. I Math., 333(6):589-592, 2001.

Martin J. Gander and Laurence Halpern. Absorbing boundary conditions for the wave equation and parallel computing. Math. Comp., 74(249):153-176 (electronic), 2005.

Martin J. Gander and Andrew M. Stuart. Space-time continuous analysis of waveform relaxation for the heat equation. SIAM J. Sci. Comput., 19(6): 2014-2031, 1998.

Eldar Giladi and Herbert B. Keller. Space-time domain decomposition for parabolic problems. Numer. Math., 93(2):279-313, 2002.

Ekachai Lelarasmee, Albert E. Ruehli, and Alberto L. SangiovanniVincentelli. The waveform relaxation method for time-domain analysis of large scale integrated circuits. IEEE Transaction on Computer-aided Design of Integrated Cicuits and Systems, CAD-1(3):131-145, 1982.

Jeffrey Rauch. Precise finite speed with bare hands. Methods Appl. Anal., 12(3):267-277, 2005.

Walter Rudin. Functional analysis. McGraw-Hill series in higher mathematics. McGraw-Hill Inc., 1973.

H. A. Schwarz. Über einen Grenzübergang durch alternierendes Verfahren. Vierteljahrsschrift der Naturforschenden Gesellschaft in Zürich, 15:272286,1870 . 